PPPL-3449

PPPL-3449

UC-70

Effects of Axial Momentum Spread on the Electron-ion

Two-stream Instability in High-intensity Ion Beams

by

Ronald C. Davidson and Hong Qin

June 2000

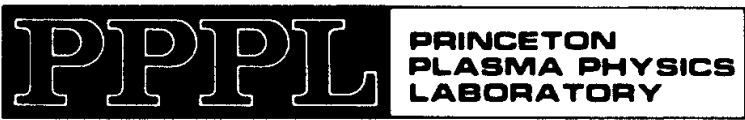

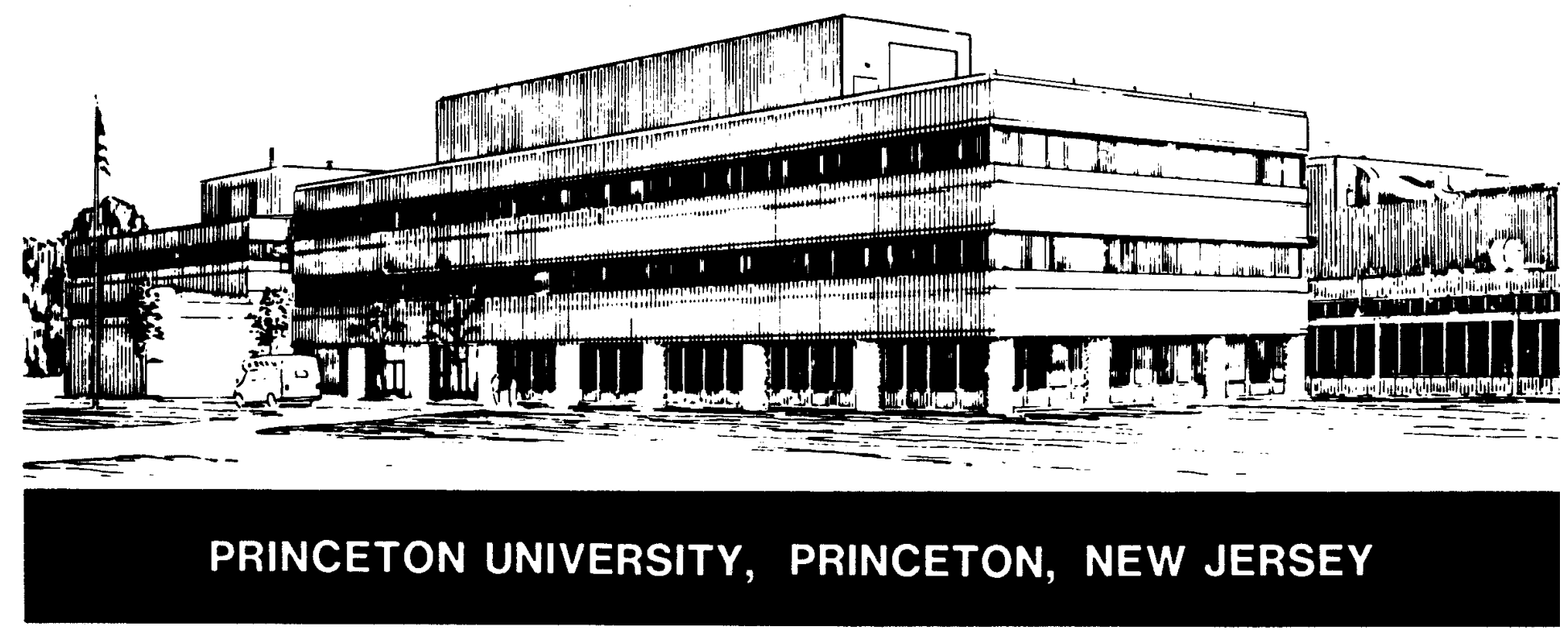




\section{PPPL Reports Disclaimer}

This report was prepared as an account of work sponsored by an agency of the United States Government. Neither the United States Government nor any agency thereof, nor any of their employees, makes any warranty, express or implied, or assumes any legal liability or responsibility for the accuracy, completeness, or usefulness of any information, apparatus, product, or process disclosed, or represents that its use would not infringe privately owned rights. Reference herein to any specific commercial product, process, or service by trade name, trademark, manufacturer, or otherwise, does not necessarily constitute or imply its endorsement, recommendation, or favoring by the United States Government or any agency thereof. The views and opinions of authors expressed herein do not necessarily state or reflect those of the United States Government or any agency thereof.

\section{Availability}

This report is posted on the U.S. Department of Energy's Princeton Plasma Physics Laboratory Publications and Reports web site in Calendar Year 2000. The home page for PPPL Reports and Publications is: http://www.pppl.gov/pub_report/

DOE and DOE Contractors can obtain copies of this report from:

U.S. Department of Energy

Office of Scientific and Technical Information

DOE Technical Information Services (DTIS)

P.O. Box 62

Oak Ridge, TN 37831

Telephone: (865) 576-8401

Fax: (865) 576-5728

Email: reports@adonis.osti.gov

This report is available to the general public from:

National Technical Information Service

U.S. Department of Commerce

5285 Port Royal Road

Springfield, VA 22161

Telephone: $1-800-553-6847$ or

(703) $605-6000$

Fax: (703) 321-8547

Internet: http://www.ntis.gov/ordering.htm 


\title{
Effects of Axial Momentum Spread on the Electron-Ion Two-Stream Instability In High-Intensity Ion Beams
}

\author{
Ronald C. Davidson and Hong Qin \\ Plasma Physics Laboratory \\ Princeton University \\ Princeton, New Jersey, 08543 USA
}

\begin{abstract}
Use is made of the Vlasov-Maxwell equations to describe the electron-ion two-stream instability driven by the directed axial motion of a high-intensity ion beam propagating through a stationary population of (unwanted) background electrons. The ion beam is treated as continuous in the z-direction, and the electrons are electrostatically confined in the transverse direction by the space-charge potential produced by the excess ion charge. The analysis is carried out for arbitrary beam intensity, consistent with transverse confinement of the beam particles, and arbitrary fractional charge neutralization by the background electrons. For the case of overlapping step-function ion and electron density profiles, corresponding to monoenergetic electrons and ions in the transverse direction, detailed stability properties are calculated, including the important effects of an axial momentum spread, over a wide range of system parameters for dipole perturbations with azimuthal mode number $\ell=1$. The two-stream instability growth rate is found to increase with increasing beam intensity, increasing fractional charge neutralization, and decreasing proximity of the conducting wall. It is shown that Landau damping associated with a modest axial momentum spread of the beam ions and background electrons has a strong stabilizing influence on the instability.
\end{abstract}


Periodic focusing accelerators and transport systems ${ }^{1-4}$ have a wide range of applications ranging from basic scientific research, to applications such as spallation neutron sources, tritium production, nuclear waste transmutation, and heavy ion fusion. At the high beam currents and charge densities of practical interest, it is increasingly important to develop an improved theoretical understanding of the influence of the intense self fields produced by the beam space charge and current on detailed equilibrium, stability and transport properties. For a one-component high-intensity beam, considerable progress has been made in describing the self-consistent evolution of the beam distribution function $f_{b}(\mathbf{x}, \mathbf{p}, t)$ and the self-generated electric and magnetic fields in kinetic analyses ${ }^{5-9}$ based on the VlasovMaxwell equations. In many practical accelerator applications, however, an (unwanted) second charge component is present. For example, a background population of electrons can result by secondary emission when energetic particles strike the chamber wall, or through ionization of background neutral gas by the beam ions. When a second charge component is present, it has been recognized for many years, both in theoretical studies and in experimental observations ${ }^{10-21}$, that the relative streaming motion of the high-intensity beam particles through the background charge species provides the free energy to drive the classical two-stream instability, appropriately modified to include the effects of dc space charge, relativistic kinematics, transverse beam dynamics, presence of a conducting wall, etc. A well-documented example is the electron-proton (e-p) instability observed in the Proton Storage Ring ${ }^{16-18}$, although a similar instability also exists for other ion species, including (for example) electron-ion interactions in electron storage rings ${ }^{19-21}$.

In a recent theoretical calculation ${ }^{10,11}$ that focuses on the moderate-intensity ion beams characteristic of proton linacs and storage rings, we developed a detailed kinetic description of the electron-ion two-stream instability based on the Vlasov-Maxwell equations. While that analysis ${ }^{10,11}$ incorporated the effects of finite transverse geometry and transverse kinetic effects, it neglected the (stabilizing) influence of an axial momentum spread of the interacting charge components. In this paper, building on the techniques developed in this earlier work $^{10,11}$, we examine two-stream stability properties, incorporating the important effects 
of an axial momentum spread on detailed stability behavior.

The present analysis considers a high-intensity ion beam with distribution function $f_{b}(\mathbf{x}, \mathbf{p}, t)$, and characteristic radius $r_{b}$ and average axial momentum $\gamma_{b} m_{b} \beta_{b} c$ propagating in the $z$-direction through a background population of electrons with distribution function $f_{e}(\mathbf{x}, \mathbf{p}, t)$. The ions have high directed axial velocity $V_{b}=\beta_{b} c$ in the $z$-direction, and the background electrons are assumed to be nonrelativistic and approximately stationary with zero average axial velocity, $\int d^{3} p v_{z} f_{e} \simeq 0$ in the laboratory frame. In the smooth-beam approximation, the ion beam is assumed to be continuous in the $z$-direction, and the applied transverse focusing force on a beam ion is modeled by

$$
\mathbf{F}_{f o c}^{b}=-\gamma_{b} m_{b} \omega_{\beta b}^{2}\left(x \hat{\mathbf{e}}_{x}+y \hat{\mathbf{e}}_{y}\right)
$$

where $\mathbf{x}_{\perp}=x \hat{\mathbf{e}}_{x}+y \hat{\mathbf{e}}_{y}$ is the transverse displacement from the beam axis, $\left(\gamma_{b}-1\right) m_{b} c^{2}$ is the characteristic directed ion kinetic energy, $m_{b}$ is the ion rest mass, $c$ is the speed of light in vacuo, and $\omega_{\beta b}=$ const. is the effective betatron frequency for transverse ion motion in the applied focusing field. For the background electrons, assuming that the ion density exceeds the background electron density, the space-charge force on an electron, $\mathbf{F}_{e}^{s}=e \nabla_{\perp} \phi$, provides transverse confinement of the background electrons by the electrostatic space-charge potential $\phi(\mathbf{x}, t)$. It is further assumed that the ion motion in the beam frame is nonrelativistic, and that the transverse momentum components of the beam ions and the characteristic spread in axial momentum are small compared with the directed axial momentum. The space-charge intensity in the present analysis is allowed to be arbitrarily large, subject only to transverse confinement of the beam ions by the focusing force in Eq. (1). Finally, the present analysis is carried out in the electrostatic and magnetostatic approximations, and the self-generated electric and magnetic fields are represented as $\mathbf{E}^{s}=-\nabla \phi(\mathbf{x}, t)$ and $\mathbf{B}^{s}=\nabla A_{z}(\mathbf{x}, t) \times \hat{\mathbf{e}}_{z}$, where the electrostatic potential $\phi(\mathbf{x}, t)$ is determined self-consistently from Poisson's equation. Treating the axial velocity profile of the beam ions as approximately uniform over the beam cross section, $V_{z b}(\mathbf{x}, t) \simeq \beta_{b} c=$ const., and assuming that the electrons carry zero axial current in the laboratory frame, the z-component of vector 
potential $A_{z}(\mathbf{x}, t)$ is determined self-consistently in the magnetostatic approximation from $\nabla^{2} A_{z}=-4 \pi Z_{b} e \beta_{b} n_{b}$. Here, $+Z_{b} e$ is the ion charge, and $n_{b}(\mathbf{x}, t)=\int d^{3} p f_{b}(\mathbf{x}, \mathbf{p}, t)$ is the ion number density.

Making use of the assumptions outlined above, collective interactions between the beam ions and the background electrons are described by the nonlinear Vlasov-Maxwell equations for the ion and electron distribution functions, $f_{b}(\mathbf{x}, \mathbf{p}, t)$ and $f_{e}(\mathbf{x}, \mathbf{p}, t)$, the space-charge potential $\phi(\mathbf{x}, t)$, and the combined potential $\psi(\mathbf{x}, t)=\phi(\mathbf{x}, t)-\beta_{b} A_{z}(\mathbf{x}, t)$. We obtain ${ }^{10,11}$

$$
\begin{gathered}
\left\{\frac{\partial}{\partial t}+\mathbf{v} \cdot \frac{\partial}{\partial \mathbf{x}}-\left(\gamma_{b} m_{b} \omega_{\beta b}^{2} \mathbf{x}_{\perp}+Z_{b} e \nabla_{\perp} \psi\right) \cdot \frac{\partial}{\partial \mathbf{p}_{\perp}}-Z_{b} e \frac{\partial \phi}{\partial z} \frac{\partial}{\partial p_{z}}\right\} f_{b}=0 \\
\left\{\frac{\partial}{\partial t}+\mathbf{v} \cdot \frac{\partial}{\partial \mathbf{x}}+e \nabla \phi \cdot \frac{\partial}{\partial \mathbf{p}}\right\} f_{e}=0 \\
\nabla^{2} \phi=-4 \pi e\left(Z_{b} \int d^{3} p f_{b}-\int d^{3} p f_{e}\right) \\
\nabla^{2} \psi=-4 \pi e\left(\frac{Z_{b}}{\gamma_{b}^{2}} \int d^{3} p f_{b}-\int d^{3} p f_{e}\right)
\end{gathered}
$$

where $\mathbf{v}=\mathbf{p} / \gamma_{b} m_{b}$ in Eq. (2), and $\mathbf{v}=\mathbf{p} / m_{e}$ in Eq. (3).

Equations (2)-(5) constitute a complete description of the collective interaction of the beam ions with the background electrons based on the Vlasov-Maxwell equations. In the present analysis, we further assume that the beam propagates axially through a perfectly conducting cylindrical pipe with radius $r=r_{w}$. Enforcing $\left[E_{\theta}^{s}\right]_{r=r_{w}}=\left[E_{z}^{s}\right]_{r=r_{w}}=\left[B_{r=r_{w}}^{s}\right]_{r}=$ 0 readily gives $\phi\left(r=r_{w}, \theta, z, t\right)=0$, and $\psi\left(r=r_{w}, \theta, z, t\right)=0$. Here, the constant values of the potentials at $r=r_{w}$ have been taken equal to zero.

Finally, under equilibrium conditions $(\partial / \partial t=0)$, the analysis assumes that ion and electron properties are spatially uniform in the $z$-direction with $\partial / \partial z=0$. However, the stability analysis assumes small-amplitude perturbations with $z$ - and $t$-variations proportional to $\exp \left(i k_{z} z-i \omega t\right)$, where $k_{z}$ is the axial wavenumber, and $\omega$ is the (complex) oscillation frequency, with $\operatorname{Im} \omega>0$ corresponding to instability. For present purposes, the stability 
analysis assumes perturbations with sufficiently long axial wavelength and high frequency that

$$
k_{z}^{2} r_{b}^{2} \ll 1
$$

where $r_{b}$ is the characteristic beam radius. Consistent with Eq. (6), we approximate $\nabla^{2} \simeq \nabla_{\perp}^{2}=\partial^{2} / \partial x^{2}+\partial^{2} / \partial y^{2}$ in Eqs. (4) and (5), and neglect to leading order the contributions proportional to $(\partial / \partial z) \delta \phi$ in the linearized versions of Eqs. (2) and (3). Our previous investigations of the electron-ion two-stream instability ${ }^{10,11}$ were carried out in the limit of cold beam ions and background electrons in the axial direction, assuming that the phase velocity, $\omega / k_{z}$, of the wave excitations satisfies $\left|\omega / k_{z}-\beta_{b} c\right| \gg v_{T b z}$, and $\left|\omega / k_{z}\right| \gg v_{T e z}$. where $v_{T b z}=\left(2 T_{b z} / \gamma_{b} m_{b}\right)^{1 / 2}$ and $v_{T e z}=\left(2 T_{e z} / m_{e}\right)^{1 / 2}$ are the characteristic axial thermal speeds of the beam ions and the background electrons, respectively. An important feature of the present analysis is the incorporation of the effects of a (small) axial momentum spread on detailed stability behavior.

Under quasisteady equilibrium conditions with $\partial / \partial t=0$, we assume axisymmetric beam propagation $(\partial / \partial \theta=0)$ and negligible variation with axial coordinate $(\partial / \partial z=0)$. It is readily shown from Eqs. (2)-(5) that the equilibrium distribution functions $(\partial / \partial t=0)$ for the beam ions and background electrons are of the general form

$$
\begin{gathered}
f_{b}^{0}(r, \mathbf{p})=F_{b}\left(H_{\perp b}\right) G_{b}\left(p_{z}\right), \\
f_{e}^{0}(r, \mathbf{p})=F_{e}\left(H_{\perp b}\right) G_{e}\left(p_{z}\right),
\end{gathered}
$$

where $r=\left(x^{2}+y^{2}\right)^{1 / 2}$ is the radial distance from the beam axis, the distributions in axial momentum are normalized according to $\int_{-\infty}^{\infty} d p_{z} G_{j}\left(p_{z}\right)=1$, for $j=b, e$, and $H_{\perp b}$ and $H_{\perp e}$ are the single-particle Hamiltonians defined by

$$
\begin{aligned}
& H_{\perp b}=\frac{1}{2 \gamma_{b} m_{b}} \mathbf{p}_{\perp}^{2}+\frac{1}{2} \gamma_{b} m_{b} \omega_{\beta b}^{2} r^{2}+Z_{b} e\left[\psi^{0}(r)-\hat{\psi}^{0}\right] \\
& H_{\perp e}=\frac{1}{2 m_{e}} \mathbf{p}_{\perp}^{2}-e\left[\phi^{0}(r)-\hat{\phi}^{0}\right] .
\end{aligned}
$$


Here, for $\partial / \partial \theta=0=\partial / \partial z, H_{\perp b}$ and $H_{\perp e}$ are exact single-particle constants of the motion in the equilibrium field configuration, and the constants $\hat{\psi}^{0} \equiv \psi^{0}(r=0)$ and $\hat{\phi}^{0} \equiv \phi^{0}(r=0)$ are the on-axis $(r=0)$ values.

There is wide latitude in specifying the functional forms of the equilibrium distribution functions ${ }^{9-11}$. Once $F_{b}\left(H_{\perp b}\right)$ and $F_{e}\left(H_{\perp e}\right)$ are specified, however, the equilibrium self-field potentials and density profiles can be calculated self-consistently from Eqs. (4) and (5) with $\partial / \partial \theta=0=\partial / \partial z$. For our purposes here, we specialize to the case of monoenergetic electrons and ions ${ }^{1,10,11,22}$

$$
\begin{aligned}
& F_{b}\left(H_{\perp b}\right)=\frac{\hat{n}_{b}}{2 \pi \gamma_{b} m_{b}} \delta\left(H_{\perp b}-\hat{T}_{\perp b}\right), \\
& F_{e}\left(H_{\perp e}\right)=\frac{\hat{n}_{e}}{2 \pi m_{e}} \delta\left(H_{\perp e}-\hat{T}_{\perp e}\right) .
\end{aligned}
$$

In this case, it is found that the density profiles $n_{j}^{0}(r), j=b, e$, correspond to overlapping step-function profiles. Here, $\hat{n}_{b}$ and $\hat{n}_{e} \equiv f Z_{b} \hat{n}_{b}$ are positive constants corresponding to the ion and electron densities, $f=$ const. is the fractional charge neutralization, and $\hat{T}_{\perp b}$ and $\hat{T}_{\perp e}$ are constants corresponding to the on-axis $(r=0)$ values of the transverse ion and electron temperatures, respectively. Without presenting algebraic details ${ }^{10,11}$, some algebraic manipulation of Eqs. (4), (5), and (7)-(9) gives the step-function density profiles $n_{j}^{0}(r)=\hat{n}_{j}=$ const., for $0 \leq r<r_{b}$, and $n_{j}^{0}(r)=0$ for $r_{b}<r \leq r_{w}$, and $j=b, e$. Here, the beam radius $r_{b}$ is related to other equilibrium parameters by $\hat{\nu}_{b}^{2} r_{b}^{2}=2 \hat{T}_{\perp b} / \gamma_{b} m_{b}$ and $\hat{\nu}_{e}^{2} r_{b}^{2}=2 \hat{T}_{\perp e} / m_{e}$, where for monoenergetic ions and electrons, the (depressed) betatron frequencies $\hat{\nu}_{b}$ and $\hat{\nu}_{e}$ are defined by

$$
\begin{aligned}
& \hat{\nu}_{b}^{2}=\omega_{\beta b}^{2}-\frac{1}{2}\left(\frac{1}{\gamma_{b}^{2}}-f\right) \hat{\omega}_{p b}^{2}=\text { const. } \\
& \hat{\nu}_{e}^{2}=\frac{1}{2} \frac{\gamma_{b} m_{b}}{Z_{b} m_{e}}(1-f) \hat{\omega}_{p b}^{2}=\text { const. }
\end{aligned}
$$

where $\hat{\omega}_{p b}^{2}=4 \pi \hat{n}_{b} Z_{b}^{2} e^{2} / \gamma_{b} m_{b}$ is the ion plasma frequency-squared. The inequalities, $\hat{\nu}_{b}^{2}>$ 0 and $\hat{\nu}_{e}^{2}>0$, are required for existence of the equilibrium. Therefore, we obtain the 
inequalities $\left(\hat{\omega}_{p b}^{2} / \omega_{\beta b}^{2}\right)\left(1-\gamma_{b}^{2} f\right)<2 \gamma_{b}^{2}$ and $f<1$, as restrictions on beam intensity and fractional charge neutralization for transverse confinement of the ions and electrons.

For small-amplitude perturbations about general equilibrium distributions, $F_{j}\left(H_{\perp j}\right)$ and $G_{j}\left(p_{z}\right), j=b, e$, and corresponding self-field potentials, $\psi^{0}(r)$ and $\phi^{0}(r)$, a stability analysis proceeds by linearizing Eqs. (2)-(5). Perturbed quantities are expressed as $\delta \psi(\mathbf{x}, t)=\delta \hat{\psi}\left(\mathbf{x}_{\perp}\right) \exp \left(i k_{z} z-i \omega t\right), \delta f_{b}(\mathbf{x}, \mathbf{p}, t)=\delta \hat{f}_{b}\left(\mathbf{x}_{\perp}, \mathbf{p}\right) \exp \left(i k_{z} z-i \omega t\right)$, etc., where $\mathbf{x}_{\perp}=(x, y)$, and $\operatorname{Im} \omega>0$ is assumed, corresponding to instability (temporal growth). Here, $k_{z}=2 \pi n / L$ is the axial wavenumber, where $n$ is an integer, and $L$ is the fundamental axial periodicity length ( $L=2 \pi R$ for a ring, where $R$ is the ring radius). The linearized Vlasov equations are formally integrated by using the method of characteristics ${ }^{1,10,23}$ to integrate along the unperturbed trajectories, $\mathbf{x}_{\perp}^{\prime}\left(t^{\prime}\right)$ and $\mathbf{p}_{\perp}^{\prime}\left(t^{\prime}\right)$, in the equilibrium field configuration. Some straightforward algebra that makes use of Eqs. (2)-(5) and the assumptions enumerated earlier gives

$$
\begin{aligned}
& \delta \hat{f}_{e}\left(\mathbf{x}_{\perp}, \mathbf{p}\right)=-e \frac{\partial}{\partial H_{\perp e}} F_{e}\left(H_{\perp e}\right)\left\{\delta \hat{\phi}\left(\mathbf{x}_{\perp}\right)+i\left(\omega-k_{z} v_{z}\right) \int_{-\infty}^{0} d \tau \delta \hat{\phi}\left(\mathbf{x}_{\perp}^{\prime}\right) \exp \left[-i\left(\omega-k_{z} v_{z}\right) \tau\right]\right\} G_{e}\left(p_{z}\right) \\
& \delta \hat{f}_{b}\left(\mathbf{x}_{\perp}, \mathbf{p}\right)=Z_{b} e \frac{\partial}{\partial H_{\perp b}} F_{b}\left(H_{\perp b}\right)\left\{\delta \hat{\psi}\left(\mathbf{x}_{\perp}\right)+i\left(\omega-k_{z} v_{z}\right) \int_{-\infty}^{0} d \tau \delta \hat{\psi}\left(\mathbf{x}_{\perp}^{\prime}\right) \exp \left[-i\left(\omega-k_{z} v_{z}\right) \tau\right]\right\} G_{b}\left(p_{z}\right)
\end{aligned}
$$

where $v_{z}=p_{z} / m_{e}$ in Eq. (11), $v_{z}=p_{z} / \gamma_{b} m_{b}$ in Eq. (12), and the potential amplitudes, $\delta \hat{\phi}\left(\mathbf{x}_{\perp}\right)$ and $\delta \hat{\psi}\left(\mathbf{x}_{\perp}\right)$, solve

$$
\begin{gathered}
\left(\frac{\partial^{2}}{\partial x^{2}}+\frac{\partial^{2}}{\partial y^{2}}\right) \delta \hat{\phi}=-4 \pi e\left[Z_{b} \int d^{3} p \delta \hat{f}_{b}-\int d^{3} p \delta \hat{f}_{e}\right] \\
\left(\frac{\partial^{2}}{\partial x^{2}}+\frac{\partial^{2}}{\partial y^{2}}\right) \delta \hat{\psi}=-4 \pi e\left[\frac{1}{\gamma_{b}^{2}} Z_{b} \int d^{3} p \delta \hat{f}_{b}-\int d^{3} p \delta \hat{f}_{e}\right]
\end{gathered}
$$

In Eqs. (11) and (12), $\tau=t^{\prime}-t$ is the displaced time variable, and the 'primed' orbits, $\mathbf{x}_{\perp}^{\prime}\left(t^{\prime}\right)$ and $\mathbf{p}_{\perp}^{\prime}\left(t^{\prime}\right)$, in the equilibrium field configuration are assumed ${ }^{10,11}$ to pass through the phase-space point $\left(\mathbf{x}_{\perp}, \mathbf{p}_{\perp}\right)$ at time $t^{\prime}=t$. 
The kinetic eigenvalue equations (11)-(14) have a wide range of applicability, and can be used to determine the complex oscillation frequency $\omega$ and detailed stability properties for a wide range of system parameters and choices of equilibrium distribution functions $F_{j}\left(H_{\perp j}\right)$ and $G_{j}\left(p_{z}\right), j=b, e$. The principal challenge in analyzing Eqs. (11)-(14) is twofold. First, depending on the equilibrium profiles, the transverse orbits $\mathbf{x}_{\perp}^{\prime}\left(t^{\prime}\right)$ are often difficult to calculate in closed analytical form. Second, once the orbits in the equilibrium fields are determined, the integrations over $t^{\prime}$ in Eqs. (11) and (12) are challenging because the orbits occur explicitly in the arguments of the (yet unknown) eigenfunction amplitudes $\delta \hat{\phi}\left(\mathbf{x}_{\perp}^{\prime}\right)$ and $\delta \hat{\psi}\left(\mathbf{x}_{\perp}^{\prime}\right)$.

For present purposes, we specialize to the choice of monoenergetic ion and electron distributions in Eq. (9), and the corresponding step-function equilibrium density profiles with $n_{j}^{0}(r)=\hat{n}_{j}=$ const., for $0 \leq r<r_{b}$, and $n_{j}^{0}(r)=0$, for $r_{b}<r \leq r_{w}$. In this case, the transverse ion orbit equation for $\mathbf{x}_{\perp}^{\prime}\left(t^{\prime}\right)$ can be integrated exactly to give

$$
\mathbf{x}_{\perp}^{\prime}\left(t^{\prime}\right)=\mathbf{x}_{\perp} \cos \left(\hat{\nu}_{b} \tau\right)+\frac{1}{\gamma_{b} m_{b} \hat{\nu}_{b}} \mathbf{p}_{\perp} \sin \left(\hat{\nu}_{b} \tau\right)
$$

for $0 \leq r^{\prime}\left(t^{\prime}\right)<r_{b}$, and the axial orbit is $z^{\prime}\left(t^{\prime}\right)=z+\left(p_{z} / \gamma_{b} m_{b}\right) \tau$. Here, $\tau=t^{\prime}-t$ is the displaced time variable, and $\hat{\nu}_{b}=$ const. is the (depressed) betatron frequency defined in Eq. (10). The electron orbit $\mathbf{x}_{\perp}^{\prime}\left(t^{\prime}\right)$ is identical in form to Eq. (15), provided we make the replacements $\gamma_{b} m_{b} \rightarrow m_{e}$ and $\hat{\nu}_{b} \rightarrow \hat{\nu}_{e}$ in Eq. (15). A careful examination of the eigenvalue equations (11)-(14) for the choice of equilibrium distributions in Eq. (9) ${ }^{10,11}$ shows that Eqs. (11)-(14) support a class of exact solutions in which the perturbed potentials have the forms, $\delta \hat{\psi}\left(\mathbf{x}_{\perp}\right)=\delta \hat{\psi}_{\ell}(r) \exp (i \ell \theta)=\hat{\psi}_{\ell} r^{\ell} \exp (i \ell \theta)$, and $\delta \hat{\phi}\left(\mathbf{x}_{\perp}\right)=\delta \hat{\phi}_{\ell}(r) \exp (i \ell \theta)=$ $\hat{\phi}_{\ell} r^{\ell} \exp (i \ell \theta)$, in the beam interior $\left(0 \leq r<r_{b}\right)$. Here, $\hat{\psi}_{\ell}$ and $\hat{\phi}_{\ell}$ are constant amplitudes, $\ell$ is the azimuthal mode number, and we have introduced cylindrical polar coordinates $(r, \theta)$ defined by $x=r \cos \theta$ and $y=r \sin \theta$. This class of exact solutions corresponds to surfacewave perturbations in which the perturbed density, $\delta \hat{n}_{j}=\int d^{3} p \delta f_{j}, j=b, e$, is localized at the beam surface $\left(r=r_{b}\right)$. What is most remarkable is that the orbit integrals over terms proportional to $r^{\prime \ell} \exp \left(i \ell \theta^{\prime}\right)=\left[x^{\prime}\left(t^{\prime}\right)+i y^{\prime}\left(t^{\prime}\right)\right]^{\ell}$ occurring in Eqs. (11) and (12) can be 
evaluated in closed analytical form, and Maxwell's equations (13) and (14) solved exactly inside $\left(0 \leq r<r_{b}\right)$ and outside $\left(r_{b}<r \leq r_{w}\right)$ the beam ${ }^{10,11}$. Enforcing the appropriate boundary conditions on $\delta \hat{\phi}_{\ell}(r)$ and $\delta \hat{\psi}_{\ell}(r)$ at $r=r_{b}$ and $r=r_{w}$ then gives a closed dispersion relation for the complex eigenfrequency $\omega$.

Derivation of the kinetic dispersion relation from Eqs. (11)-(14) closely parallels the analysis in Ref. 10 and 11. Without presenting algebraic details, for perturbations with azimuthal mode number $\ell$ and axial wavenumber $k_{z}$, we obtain the dispersion relation

$$
\begin{aligned}
{\left[\frac{2}{1-\left(r_{b} / r_{w}\right)^{2 \ell}}\right.} & \left.+\frac{\hat{\omega}_{p b}^{2}}{\ell \gamma_{b}^{2} \hat{\nu}_{b}^{2}} \Gamma_{b}^{\ell}(\omega)\right]\left[\frac{2}{1-\left(r_{b} / r_{w}\right)^{2 \ell}}+\frac{\hat{\omega}_{p e}^{2}}{\ell \hat{\nu}_{e}^{2}} \Gamma_{e}^{\ell}(\omega)\right] \\
& =\frac{\hat{\omega}_{p e}^{2}}{\ell \hat{\nu}_{e}^{2}} \cdot \frac{\hat{\omega}_{p b}^{2}}{\ell \hat{\nu}_{b}^{2}} \Gamma_{e}^{\ell}(\omega) \Gamma_{b}^{\ell}(\omega)
\end{aligned}
$$

where $\hat{\omega}_{p e}^{2}=4 \pi \hat{n}_{e} e^{2} / m_{e}, \hat{\omega}_{p b}^{2}=4 \pi \hat{n}_{b} Z_{b}^{2} e^{2} / \gamma_{b} m_{b}$, and $\hat{\nu}_{b}$ and $\hat{\nu}_{e}$ are the (depressed) betatron frequencies defined in Eq. (10). The ion and electron susceptibilities, $\Gamma_{j}^{\ell}(\omega), j=b, e$, occurring in Eq. (16) are defined by

$$
\Gamma_{j}^{\ell}(\omega)=-\frac{1}{2^{\ell}} \sum_{m=0}^{\ell} \frac{\ell !}{m !(\ell-m) !} \int_{-\infty}^{\infty} d p_{z} \frac{(\ell-2 m) \hat{\nu}_{j} G_{j}\left(p_{z}\right)}{\left[\left(\omega-k_{z} v_{z}\right)-(\ell-2 m) \hat{\nu}_{j}\right]},
$$

for general azimuthal mode number $\ell$, and (yet unspecified) distribution in axial momentum $G_{j}\left(p_{z}\right)$. In carrying out the integration over $p_{z}$ in Eq. (17), Imw $>0$ is assumed ${ }^{23,24}$.

Equation (16) is the final form of the kinetic dispersion relation, derived from the linearized Vlasov-Maxwell equations for small-amplitude perturbations about the monoenergetic equilibrium distributions in Eq. (9) and the corresponding step-function density profiles. As such, Eq. (16) can be used to determine the complex oscillation frequency $\omega$ over a wide range of system parameters, including normalized beam intensity $\left(\hat{\omega}_{p b}^{2} / 2 \gamma_{b}^{2} \omega_{\beta b}^{2}\right)$, fractional charge neutralization $\left(f=\hat{n}_{e} / Z_{b} \hat{n}_{b}\right)$, azimuthal mode number $(\ell)$, axial wavenumber $\left(k_{z}\right)$, choice of $G_{j}\left(p_{z}\right)$, etc., subject only to the simplifying assumptions summarized earlier in this paper. In the absence of electrons $\left(\hat{n}_{e}=0\right)$, the dispersion relation (16) supports stable collective oscillations of the ion beam, and reveals a rich harmonic content at frequencies $\omega-k_{z} V_{b} \simeq \pm \hat{\nu}_{b}, \pm 2 \hat{\nu}_{b}, \cdots, \pm \ell \hat{\nu}_{b}$. When background electrons are present $\left(\hat{n}_{e} \neq 0\right)$, 
however, Eq. (16) supports unstable solutions ( $\operatorname{Im} \omega>0)$ with instability resulting from the axial streaming $\left(V_{b} \neq 0\right)$ of the beam ions through the background electrons, at least in the limit where the ion and electron axial motions are 'cold' ${ }^{10,11}$, with $G_{b}\left(p_{z}\right)=\delta\left(p_{z}-\gamma_{b} m_{b} V_{b}\right)$ and $G_{e}\left(p_{z}\right)=\delta\left(p_{z}\right)$.

The $p_{z}$-integration in Eq. (17) can be carried out for a variety of choices of $G_{j}\left(p_{z}\right)$ ranging from a shifted Maxwellian, to a step-function distribution, to a Lorentzian distribution. For analytical simplicity, we consider here the case of Lorentzian distributions with

$$
\begin{aligned}
G_{b}\left(p_{z}\right) & =\frac{\Delta_{b}}{\pi\left[\left(p_{z}-\gamma_{b} m_{b} V_{b}\right)^{2}+\Delta_{b}^{2}\right]}, \\
G_{e}\left(p_{z}\right) & =\frac{\Delta_{e}}{\pi\left(p_{z}^{2}+\Delta_{e}^{2}\right)},
\end{aligned}
$$

where $\Delta_{j}=$ const. $>0$ is a measure of the axial momentum spread, and $p_{z}$ and $v_{z}$ are related by $p_{z}=m_{e} v_{z}$ for the electrons, and $p_{z}=\gamma_{b} m_{b} v_{z}$ for beam the ions. Note from Eq. (18) that $V_{b}=\int_{-\infty}^{\infty} d p_{z} v_{z} G_{b}\left(p_{z}\right)$, and $0=\int_{-\infty}^{\infty} d p_{z} v_{z} G_{e}\left(p_{z}\right)$, which corresponds to the beam ions streaming axially through a stationary electron background. Substituting Eq. (18) into Eq. (17) and integrating over $p_{z}$ for $\operatorname{Im} \omega>0$ readily gives the simple expression

$$
\Gamma_{j}^{\ell}(\omega)=-\frac{1}{2^{\ell}} \sum_{m=0}^{\ell} \frac{\ell !}{m !(\ell-m) !} \frac{(\ell-2 m) \hat{\nu}_{j}}{\left[\left(\omega-k_{z} V_{j}+i\left|k_{z}\right| v_{T j z}\right)-(\ell-2 m) \hat{\nu}_{j}\right]} .
$$

Here, $V_{e}=0$ for the electrons, and $v_{T j z}$ is a measure of the characteristic axial thermal speed, defined by $v_{T b z}=\Delta_{b} / \gamma_{b} m_{b}$ for the beam ions, and $v_{T e z}=\Delta_{e} / m_{e}$ for the background electrons. Substituting Eq. (19) into Eq. (16), the resulting dispersion relation can be used to investigate the effects of an axial momentum spread on detailed properties of the electronion two-stream instability for general azimuthal mode number $\ell$ over a wide range of system parameters.

A careful examination of Eq. (16) for $\hat{n}_{e} \neq 0$ shows that the strongest instability (largest growth rate) occurs for azimuthal mode number $\ell=1$, corresponding to a simple (dipole) displacement of the beam ions and the background electrons. For $\ell=1$, we substitute Eq. (19) into Eq. (16), and introduce the electron and ion collective oscillation frequencies, $\omega_{e}$ and $\omega_{b}$, defined by 


$$
\begin{gathered}
\omega_{e}^{2} \equiv \hat{\nu}_{e}^{2}+\frac{1}{2} \hat{\omega}_{p e}^{2}\left(1-\frac{r_{b}^{2}}{r_{w}^{2}}\right)=\frac{1}{2} \frac{\gamma_{b} m_{b}}{Z_{b} m_{e}} \hat{\omega}_{p b}^{2}\left(1-f \frac{r_{b}^{2}}{r_{w}^{2}}\right), \\
\omega_{b}^{2} \equiv \hat{\nu}_{b}^{2}+\frac{\hat{\omega}_{p b}^{2}}{2 \gamma_{b}^{2}}\left(1-\frac{r_{b}^{2}}{r_{w}^{2}}\right)=\omega_{\beta b}^{2}+\frac{1}{2} \hat{\omega}_{p b}^{2}\left(f-\frac{1}{\gamma_{b}^{2}} \frac{r_{b}^{2}}{r_{w}^{2}}\right),
\end{gathered}
$$

where $\hat{\omega}_{p e}^{2}$ has been expressed as $\hat{\omega}_{p e}^{2}=\left(\gamma_{b} m_{b} / Z_{b} m_{e}\right) f \hat{\omega}_{p b}^{2}$. Substituting into Eq. (16) and rearranging terms, the $\ell=1$ dispersion relation can be expressed in the compact form

$$
\left[\left(\omega-k_{z} V_{b}+i\left|k_{z}\right| v_{T b z}\right)^{2}-\omega_{b}^{2}\right]\left[\left(\omega+i\left|k_{z}\right| v_{T e z}\right)^{2}-\omega_{e}^{2}\right]=\omega_{f}^{4}
$$

where $\omega_{f}$ is defined by

$$
\omega_{f}^{4} \equiv \frac{1}{4} f\left(1-\frac{r_{b}^{2}}{r_{w}^{2}}\right)^{2} \frac{\gamma_{b} m_{b}}{Z_{b} m_{e}} \hat{\omega}_{p b}^{4}
$$

In the cold limit $\left(v_{T b z}=0=v_{T e z}\right)$, and in the absence of background electrons $(f=0$ and $\left.\omega_{f}=0\right)$, Eq. (21) gives stable collective oscillations of the ion beam with frequency $\omega-k_{z} V_{b}= \pm \omega_{b}$, where $\omega_{b}$ is defined in Eq. (20). For $f \neq 0$, however, the ion and electron terms on the left-hand side of Eq. (21) are coupled by the $\omega_{f}^{4}$ term on the right-hand side, leading to one unstable solution with $\operatorname{Im} \omega>0$ for a certain range of axial wavenumber $k_{z}$. The instability is two-stream in nature, and results from the directed ion motion with axial velocity $V_{b}$ through the (stationary) background electrons. A careful examination ${ }^{10,11}$ of Eq. (21) shows that the unstable, positive-frequency branch has frequency and wavenumber $\left(\omega, k_{z}\right)$ closely tuned to the values $\left(\omega_{0}, k_{z 0}\right)$ defined by $\omega_{0}=+\omega_{e}$ and $\omega_{0}-k_{z 0} V_{b}=-\omega_{b}$, or equivalently, $k_{z 0}=\left(\omega_{e}+\omega_{b}\right) / V_{b}$. This gives

$$
\frac{\omega_{0}}{k_{z 0}}-V_{b} \simeq-\frac{\omega_{b}}{\omega_{e}+\omega_{b}} V_{b}
$$

Because $\omega_{b} \ll \omega_{e}$ in the regimes of practical interest [Eq. (20)], it follows from Eq. (23) that the phase velocity of the unstable mode is downshifted only slightly from the directed beam velocity $V_{b}$, and could be strongly affected by Landau damping by the beam ions for modest values of $v_{T b z} / V_{b} \neq 0$.

Returning to the full dispersion relation $(21)$ for $v_{T j z} \neq 0$, it is important to recognize that Eq. (21) is applicable over a wide range of normalized beam intensity $\left(\hat{\omega}_{p b}^{2} / 2 \gamma_{b}^{2} \omega_{\beta b}^{2}\right)$ and 
fractional charge neutralization (f) consistent with $\hat{\nu}_{b}^{2}>0$ and $\hat{\nu}_{e}^{2}>0$. That is, Eq. (21) can be applied to the moderate-intensity ion beams $\left(\hat{\omega}_{p b}^{2} / 2 \gamma_{b}^{2} \omega_{\beta b}^{2} \lesssim 0.2\right.$, say) in the proton linacs and storage rings envisioned for the Spallation Neutron Source (SNS) and the Proton Storage Ring (PSR) ${ }^{16-18}$. On the other hand, Eq. (21) can also be applied to the low-emittance, very high-intensity ion beams $\left(\hat{\omega}_{p b}^{2} / 2 \gamma_{b} \omega_{\beta b}^{2} \rightarrow 1\right)$ envisioned for heavy ion fusion ${ }^{4}$.

Typical results for the unstable solution to Eq. (21) are illustrated in Fig. 1 (for $v_{T b z}=$ $\left.0=v_{T e z}\right)$ and in Fig. 2 (for $v_{T b z} \neq 0$, and $\left.v_{T b z}=v_{T e z}\right)$. The system parameters in Figs. 1 and 2 correspond to $m_{b} / m_{e}=1836$ (protons), $\left(\gamma_{b}-1\right) m_{b} c^{2}=800 \mathrm{Mev}, r_{b} / r_{w}=0.5$, and $f=1$.

Shown in Fig. 1, for $v_{T b z}=0=v_{T e z}$, are plots of $(\operatorname{Im} \omega) / \omega_{\beta b}$ and $\left(\operatorname{Re} \omega-\omega_{e}\right) / \omega_{\beta b}$ versus $\left(k_{z}-k_{z 0}\right) V_{b} / \omega_{\beta b}$, where $k_{z 0} \equiv\left(\omega_{e}+\omega_{b}\right) / V_{b}$, obtained from Eq. (21) for the unstable branch for several values of $\hat{\omega}_{p b}^{2} / 2 \gamma_{b}^{2} \omega_{\beta b}^{2}$ ranging from 0.05 to 0.5 . At low beam intensities, the instability growth rate in Fig. 1 is relatively small and has a narrow bandwidth in $k_{z}$-space, symmetric about $k_{z}=k_{z 0}$. On the other hand, as the normalized beam intensity $\hat{\omega}_{p b}^{2} / 2 \gamma_{b}^{2} \omega_{\beta b}^{2}$ is increased to 0.5 , the instability bandwidth increases significantly in Fig. 1, and the growth rate becomes substantial, with $(\operatorname{Im} \omega)_{\max } \simeq 1.2 \omega_{\beta b}$. As reported previously ${ }^{10,11}$, the twostream growth rate calculated from Eq. (21) also increases with increasing fractional charge neutralization $f$, and increasing values of $r_{w} / r_{b}$.

To illustrate the stabilizing influence of parallel kinetic effects on the two-stream instability, shown in Fig. 2 is plot of $(\operatorname{Im} \omega) / \omega_{\beta b}$ versus $\left(k_{z}-k_{z 0}\right) / \omega_{\beta b}$, obtained from Eq. (21) for the unstable branch for fixed value of the normalized beam intensity, $\hat{\omega}_{p b}^{2} / 2 \gamma_{b}^{2} \omega_{p b}^{2}=0.07$, and values of $v_{T b z} / V_{b}$ ranging from 0 to 0.01. Furthermore, for purpose of illustration, in Fig. 2 we have fixed the axial momentum spread of the electrons by the value $v_{T e z}=v_{T b z}$. Because the characteristic phase velocity of the unstable mode is downshifted only slightly from the directed beam velocity $V_{b}$ [Eq. (23)], it is expected that Landau damping by parallel kinetic effects can have a strong stabilizing influence at modest values of $v_{T b z} / V_{b}$. That this is indeed the case is evident from Fig. 2, which shows a substantial reduction in maximum growth rate and eradication of the instability over the instability bandwidth as $v_{T z b} / V_{b}$ is 
increased from 0 to 0.01 .

The dispersion relation (21) can be used to derive an analytical criterion ${ }^{25}$ for stabilization of the two-stream instability by parallel kinetic effects, valid for normalized beam intensity $s_{b} \equiv \hat{\omega}_{p b}^{2} / 2 \gamma_{b} \omega_{\beta b}^{2}$ ranging from the moderate values $\left(s_{b} \lesssim 0.2\right)$ of interest in proton machines, to the space-charge dominated beams $\left(s_{b} \rightarrow 1\right)$ of interest in heavy ion fusion. For our purpose here, we present the stability criterion for moderate values of $s_{b}=\hat{\omega}_{p b}^{2} / 2 \gamma_{b} \omega_{\beta b}^{2} \lesssim 0.2$. It is convenient to express $\delta \omega=\omega-\omega_{e}$ and $\delta k_{z}=k_{z}-k_{z 0}$, where $k_{z 0}=\left(\omega_{e}+\omega_{b}\right) / V_{b}$, and $\omega_{e}$ and $\omega_{b}$ are defined in Eq. $(20)$. For $s_{b} \ll 1$, because $|\delta \omega| \ll \omega_{b}$, we [see Figs. 1 and 2, and Eq. (20)], it follows that the dispersion relation (21) can be approximated by the quadratic form.

$$
\left(\delta \omega+i\left|k_{z}\right| v_{T b z}-\delta k_{z} V_{b}\right)\left(\delta \omega+i\left|k_{z}\right| v_{T e z}\right)=-\frac{\omega_{f}^{4}}{4 \omega_{e} \omega_{b}}
$$

for $\left(\omega, k_{z}\right)$ in the vicinity of $\left(\omega_{0}, k_{z 0}\right)$. The solution to Eq. (24) with $\gamma=\operatorname{Im}(\delta \omega)>0$ corresponds to instability (temporal growth), with maximum growth rate occurring for $\delta k_{z}=$ 0 (see Fig. 2). For cold beam ions and background electrons with $v_{T e z}=0=v_{T b z}$, setting $\delta k_{z}=0$ in Eq. (24) gives the maximum growth rate $(\operatorname{Im} \delta \omega)_{\max }=\left(\omega_{f}^{4} / 4 \omega_{e} \omega_{b}\right)^{1 / 2}$. On the other hand, as $v_{T b z}$ and $v_{T e z}$ are increased, the maximum growth rate decreases, and it can be shown from Eq. (24) that the spectrum of unstable modes is completely stabilized $(\operatorname{Im} \delta \omega<0)$ whenever $k_{z 0}^{2} v_{T b z} v_{T e z}>\omega_{f}^{4} / 4 \omega_{e} \omega_{b}$, or equivalently,

$$
\frac{v_{T b z} v_{T e z}}{V_{b}^{2}}>\frac{\omega_{f}^{4}}{4 \omega_{e} \omega_{b}\left(\omega_{e}+\omega_{b}\right)^{2}}
$$

where $\omega_{b}, \omega_{e}$, and $\omega_{f}$ are defined in Eqs. (20) and (22).

In the regimes of practical interest, the right-hand side of Eq. (25) is very small, and only modest momentum spreads are required to provide complete stabilization of the twostream instability. For example, assuming $r_{b} / r_{w} \ll 1$ and $s_{b} \ll 1$, and taking $v_{T e z}=v_{T b z}$ for purpose of illustration, Eq. (25) can be expressed as

$$
\frac{\Delta p_{z b}}{\gamma_{b} m_{b} V_{b}}>\frac{1}{2} \gamma_{b}^{1 / 2} f^{1 / 2} s_{b}^{1 / 4}\left(\frac{m_{e}}{\gamma_{b} m_{b}}\right)^{1 / 4}
$$


where $\Delta p_{z b} / \gamma_{b} m_{b} V_{b}=v_{T b z} / V_{b}, \Delta p_{z b}=\Delta_{b}$ is the axial momentum spread [see Eq. (18)], and $s_{b}=\hat{\omega}_{p b}^{2} / 2 \gamma_{b} \omega_{\beta b}^{2}$ is the normalized beam intensity. As illustrative parameters, for $f=0.1$, $s_{b}=0.07, \gamma_{b}=1.85$ and $m_{b} / m_{e}=1836$, Eq. (26) predicts complete stabilization for $\Delta p_{z b} / \gamma_{b} m_{b} V_{b}>1.4 \%$.

In conclusion, we have outlined here the derivation of the fully kinetic dispersion relation (21) describing the electron-ion two-stream instability for an intense ion beam propagating through a stationary population of background electrons. For $k_{z}^{2} r_{b}^{2} \ll 1$, the dispersion relation (21) incorporates the leading-order effects of an axial momentum spread in the ion and electron components, and can be used to investigate detailed stability properties over a wide range of normalized beam intensity $\left(\hat{\omega}_{p b}^{2} / 2 \gamma_{b}^{2} \omega_{\beta b}^{2}\right)$, fractional charge neutralization $(f=$ $\left.\hat{n}_{e} / Z_{b} \hat{n}_{b}\right)$, azimuthal mode number $(\ell)$, and axial wavenumber $\left(k_{z}\right)$. For dipole perturbations $(\ell=1)$, it has been shown that Landau damping by parallel kinetic effects can have a strong stabilizing influence on the electron-ion two-stream instability. The condition for complete stabilization of the two-stream instability by parallel kinetic effects is given by Eq. (25) (valid for moderate values of beam intensity), which corresponds to a relatively small axial momentum spread [see Eq. (26)]. If the beam ions and background electrons are initially cold axially, it is expected that a nonlinear consequence of the two-stream instability would be to cause an increase in axial momentum spread, thereby leading to a (quasilinear) stabilization of the instability.

\section{ACKNOWLEDGMENTS}

This research was supported by the U.S. Department of Energy and the Short-Pulse Spallation Source project, LANSCE Division of the Los Alamos National Laboratory, and the Spallation Neutron Source project. 


\section{REFERENCES}

${ }^{1}$ R. C. Davidson, Physics of Nonneutral Plasmas (Addison-Wesley Publishing Co., Reading, MA, 1990), and references therein.

${ }^{2}$ M. Reiser, Theory and Design of Charged Particle Beams (John Wiley \& Sons, Inc., New York, 1994).

3 T. P. Wangler, Principles of RF Linear Accelerators (John Wiley \& Sons, Inc., New York, 1998).

${ }^{4}$ See, for example, Proceedings of the 1995 International Symposium on Heavy Ion Inertial Fusion (Eds., J. J. Barnard, T. J. Fessenden, and E. P. Lee) J. Fusion Engineering and Design 32, pp. 1-620 (1996), and references therein.

${ }^{5}$ T. -S. Wang and L. Smith, Particle Accelerators 12, 247 (1982).

${ }^{6}$ J. Struckmeier and I. Hofmann, Particle Accelerators 39, 219 (1992).

${ }^{7}$ R. C. Davidson, Physics of Plasmas 5, 3459 (1998).

${ }^{8}$ R. C. Davidson, Physical Review Letters 81, 991 (1998).

${ }^{9}$ R. C. Davidson and C. Chen, Particle Accelerators 59, 175 (1998).

${ }^{10}$ R. C. Davidson, H. Qin, P. H. Stoltz, and T. -S. Wang, Physical Review Special Topics on Accelerators and Beams 2, 054401 (1999), and references therein.

${ }^{11}$ R. C. Davidson, H. Qin and T. -S. Wang, Physics Letters A252, 213 (1999).

${ }^{12}$ H. Qin, R. C. Davidson and W. W. Lee, American Institute Conference Proceedings 496, 295 (1999).

${ }^{13}$ D. G. Koshkarev and P. R. Zenkevich, Particle Accelerators 3, 1 (1972).

${ }^{14}$ E. Keil and B. Zotter, CERN Report CERN-ISR-TH/71-58 (1971).

${ }^{15}$ L. J. Laslett, A. M. Sessler, and D. Möhl, Nuclear Instruments and Methods in Physics Research 121, 517 (1974).

${ }^{16}$ D. Neuffer, E. Colton, D. Fitzgerald, T. Hardek, R. Hutson, R. Macek, M. Plum, H. 
Thiessen, and T. -S. Wang, Nuclear Instruments and Methods in Physics Research A321, 1 (1992).

${ }^{17}$ D. Neuffer and C. Ohmori, Nuclear Instruments and Methods in Physics Research A343, 390 (1994).

${ }^{18}$ R. Macek, Proceeding of the Workshop on Space Charge Physics in High Intensity Hadron Rings, American Institute Conference Proceedings 448, 116 (1998).

${ }^{19}$ M. Izawa, Y. Sato, and T. Toyomasu, Phys. Rev. Lett. 74, 5044 (1995).

${ }^{20}$ J. Byrd, A. Chao, S. Heifets, M. Minty, T. O. Raubenheimer, J. Seeman, G. Stupakov, J. Thomson and F. Zimmerman, Phys. Rev. Lett. 79, 79 (1997).

${ }^{21}$ K. Ohmi, Phys. Rev. E 55, 7550 (1997).

${ }^{22}$ I. Kapchinskij and V. Vladimirskij, in Proceedings of the International Conference on High Energy Accelerators and Instrumentation (CERN Scientific Information Service, Geneva, 1959), p. 274.

${ }^{23}$ See, for example, Chapters 2, 4, and 10 of Ref. 1.

${ }^{24}$ N. A. Krall and A. W. Trivelpiece, Principles of Plasma Physics (San Francisco Press, San Francisco, CA, 1986).

${ }^{25}$ R. C. Davidson, H. Qin, I. Kaganovich and W. Wei-li Lee, "Stabilizating Influence of Axial Momentum Spread on the Electron-Ion Two-Stream Instability in Intense Heavy Ion Beams", submitted for publication (2000). 


\section{FIGURES}

FIG. 1. Plots of (a) normalized growth rate $\operatorname{Im} \omega / \omega_{\beta b}$, and (b) normalized real frequency $\left(R e \omega-\omega_{e}\right) / \omega_{\beta b}$ versus shifted axial wavenumber $\left(k_{z}-k_{z 0}\right) V_{b} / \omega_{\beta b}$ obtained from the dispersion relation (21) for the unstable branch with positive real frequency. System parameters correspond to $v_{T b z}=0=v_{T e z}, m_{b} / m_{e}=1836$ (protons), $\left(\gamma_{b}-1\right) m_{b} c^{2}=800 \mathrm{MeV}, r_{b} / r_{w}=0.5$, and $f=0.1$. Curves are shown for several values of normalized beam intensity $\hat{\omega}_{p b}^{2} / 2 \gamma_{b}^{2} \omega_{\beta b}^{2}$ ranging from 0.05 to 0.5 .

FIG. 2. Plot of normalized growth rate $I m \omega / \omega_{\beta b}$ versus shifted axial wavenumber $\left(k_{z}-k_{z 0}\right) V_{b} / \omega_{\beta b}$ obtained from Eq. (21) for the unstable branch with positive real frequency. System parameters correspond to $\hat{\omega}_{p b}^{2} / 2 \gamma_{b}^{2} \omega_{\beta b}^{2}=0.07, v_{T e z}=v_{T b z}, m_{b} / m_{e}=1836$ (protons), $\left(\gamma_{b}-1\right) m_{b} c^{2}=800 \mathrm{MeV}, r_{b} / r_{w}=0.5$, and $f=0.1$. Curves are shown for several values of normalized ion thermal spread $v_{T b z} / V_{b}$ ranging from 0 to 0.01 . 
The Princeton Plasma Physics Laboratory is operated by Princeton University under contract with the U.S. Department of Energy.

\author{
Information Services \\ Princeton Plasma Physics Laboratory \\ P.O. Box 451 \\ Princeton, NJ 08543
}

Phone: 609-243-2750

Fax: 609-243-2751

e-mail: pppl_info@pppl.gov

Internet Address: http://www.pppl.gov 